“COLENDA POESIS, ET INDE POETE” (GEN. DEOR. GENT., XIV, 22, 11): BOCCACCIO, LA NOVITÀ DELLA LETTERATURA E LA LIBERTÀ DEI POETI

\title{
MatTeo Bosisio
}

Riassunto: L'articolo, dopo aver mostrato le esigenze storiche e culturali che spinsero intellettuali quali Mussato e Petrarca a ricodificare lo statuto della letteratura, si sofferma sulla difesa della poesia operata da Boccaccio. Il certaldese non solo giunge a un risultato innovativo, totalmente svincolato dai modelli medievali, ma, diversamente dal passato, prende in considerazione anche il problema del ruolo sociale dello scrittore e del rapporto con la committenza. Analizzando soprattutto i libri XIV e XV delle Genealogie, il contributo mette in luce le complesse metodologie e tecniche letterarie adottate per dirimere tali questioni. In particolare Boccaccio - inventandosi un committente fittizio, ricercando un pubblico dotto e avvertito, celebrando l'autonomia della poesia e sostenendo alcune nuove discipline come la lingua greca - traccia un modello di letterato indipendente e libero.

Le numerose riflessioni sorte nel corso del Trecento intorno allo statuto della poesia testimoniano il graduale passaggio da una stagione, storica e letteraria, a un'altra: dalla morte di Dante al primo fondamentale incontro tra le altre due 'corone' del 1350 trascorrono poco meno di trent'anni, tuttavia, in questo breve periodo, la realtà storica italiana si modifica radicalmente, con un ritmo sconosciuto per il Medioevo. L'Impero, infatti, con Ludovico il Bavaro (1282-1347) e Carlo IV (1316-1378) perde ogni spinta universalistica ancora latente, in favore dell'affermazione degli stati nazionali e regionali; la lenta formazione in Italia dei poteri locali accentua una fase di instabile transizione già avviata da tempo; gli orrori recati dalla peste del 1348 incidono inevitabilmente sulla mentalità collettiva e gli equilibri sociali; la crisi economica del settore tessile e bancario, le carestie, il crollo demografico e l'abbandono delle campagne arrestano l'ascesa delle classi più dinamiche, mentre deprimono la popolazione rurale; la marginalità della Chiesa - la cui sede era passata ad Avignone dal 1309 - e il declino di alcuni ordini monastici favoriscono la crescita di un sentimento religioso personale, poco legato alle istituzioni ${ }^{1}$.

1 Sulla questione della poesia si concentrano Frasso; Garin (1954); Mésoniat e Ronconi. Per un esame dei singoli temi storici accennati rinvio a Bergdolt; Cherubini; Chittolini; Penco e Romano. La vita e la produzione artistica di Boccaccio vengono inserite da Padoan entro il contesto storico delineato. 
Se è possibile lo scenario culturale, segnatamente letterario, subisce scosse ancora più profonde e destabilizzanti, poiché destinate a cambiare in modo definitivo la sensibilità precedente: la sempre più salda padronanza nella lingua latina di epoca repubblicana e imperiale, contraddistinta da un alto grado di complessità e fascino, porta alla svalutazione della mediolatinità, delle artes dictaminis e della lingua cancelleresca; gli approfonditi studi dei classici, la progressiva familiarità con il greco e le prime significative scoperte filologiche apportate dai circoli preumanistici veneti e poi da Petrarca aprono la strada a una nuova lettura del mondo antico, che miri al recupero effettivo dei testi senza le alterazioni imposte dalla tradizione simbolica cristiana e dalla trascuratezza degli scribi; le bussole del sapere filosofico, il tomismo e la scolastica, non sono più in grado di spiegare un universo molteplice e cedono perciò il passo a proposte laiche e più aperte ${ }^{2}$.

Questi decenni tumultuosi determinano così, all'interno della civiltà letteraria italiana, alcuni decisivi e obbligati mutamenti nella concezione della letteratura e nel ruolo sociale degli scrittori: ad esempio le interpretazioni allegoriche dei testi letterari, valide fino a Dante, risultavano insoddisfacenti e non più proponibili agli intellettuali delle generazioni successive, in quanto il confronto serrato con la latinità aveva posto il problema drammatico di conciliare il messaggio degli antichi con la fede 3 . Dante rie-

2 Sull'argomento mi sono avvalso di Avesani; Billanovich; Dionisotti; Gargan (1976); Vasoli e Weiss. Petrucci ha sostenuto: "Altissima è perciò la considerazione che il Petrarca riserva alla dignità dello scrivere. E con lui, forse per la prima volta in modo consapevole, non si tratta più di una dignità giustificata da simbolismo religioso, da superstizione magica o da estremo raffinamento tecnico, come nei secoli passati: bensì di un sentimento che scaturiva dalla natura stessa della funzione letteraria di creazione e di espressione del pensiero, di cui la scrittura è, e deve essere, strumento fedele" (11).

3 Si raffronti quanto sostiene Isidoro di Siviglia nelle Etimologie (VIII, 7, 10): "Officium autem poetae in eo est ut ea, quae vere gesta sunt, in alias species obliquis figurationibus cum decore aliquo conversa transducat" ("compito del poeta è trasporre in forme differenti avvenimenti reali trasformati con una qualche eleganza mediante l'uso di immagini translate"). Ugo da San Vittore nel Didascalion de studio legendi $(\mathrm{V}, 3)$ aveva affermato che per le Sacre scritture sono portatrici di significato "non tantum verba, sed etiam res", seguendo un processo per cui "per vocem ad intellectum, per intellectum ad rem, per rem ad rationem, per rationem pervenitur ad veritatem" ("non soltanto le parole, ma anche le cose"; "dalla parola al pensiero, dal pensiero alle cose, dalle cose alla mente divina e quindi alla verità"; la traduzione è mia). Inoltre la poesia - seguendo il principio del $D e$ doctrina christiana (IV, 11) di S. Agostino per cui occorre "in verbis verum amare, non verba" - non si sarebbe dovuta affrancare mai dalle finalità imprescindibi- 
sce ancora a collocare la propria civiltà — e di conseguenza l'intera operazione culturale della Commedia - come necessariamente superiore: si pensi al celebre passo del canto XXVIII del Purgatorio (vv. 139-141) in cui la conoscenza mendace del Parnaso presso i pagani viene descritta quale vago presentimento del vero cristiano, simboleggiato dall'Eden ${ }^{4}$. Per Petrarca e Boccaccio, invece, il rapporto più attento con il passato aveva fatto scoprire una letteratura valida, dotata di notevole autonomia, foriera di contenuti alti e indipendenti dalla morale cristiana nonché svincolata dalle aporie e volgarità dell'epoca presente 5 .

Pertanto, il bisogno di configurare gli scopi e le caratteristiche costitutive della poesia va a intrecciarsi con l'esigenza di ridefinire il ruolo sociale degli intellettuali: se non si garantisce più l'intimo collegamento tra il prodotto letterario e la verità religiosa, occorre trovare un diverso ubi consistam per avvalorare l'agire artistico, incalzato anche dall'avanzamento delle restanti arti liberali e di quelle meccaniche ${ }^{6}$. Oltretutto, con i primi anni del XIV secolo e l'espansione delle Signorie, si viene sempre più a perdere quel senso di utilità collettiva per la letteratura e si crea una frattura tra il compito dello scrittore e la comunità di appartenenza. A causa del distacco tra gli studi giuridici e quelli letterari — non a caso messi più volte in rapporto di insanabile conflitto da Boccaccio (cfr. ad es. Genealogie deorum gentilium, XV, 10, 6-8 e Vita di Petrarca, V) - gli intellettuali, prima vicini alle esigenze del proprio comune, si trasformano in letterati tout court che concepiscono l'attività professionale al di fuori di un impegno complessivo di ordine politico, religioso o pratico ${ }^{7}$. E, tuttavia, l'aspirazione suggerita dai classici di totale indipendenza e libertà viene subito frustrata

li dell'utilitas e dell'honestum. Per uno studio approfondito sull'allegoria rinvio a Russell (1988). Le traduzioni — laddove non venga specificato, sempre tratte dall'edizione di riferimento - sono limitate ai periodi più complessi.

${ }^{4}$ Un'efficace trattazione sull'interpretazione allegorica della cultura pagana in Dante è fornita da Pépin, secondo cui nel fiorentino "è presente una spiccata propensione a scorgere intenzioni nascoste nei versi dei suoi predecessori" (158161). Cfr. anche i recenti studi di Ascoli; Iori; Mendez; Moudarres e Spera.

5 Il topos oppositivo tra la poesia e la corruzione della contemporaneità è rintracciabile in Petrarca ( $R V F$, VII e Sen. X, 2) e Boccaccio (Rime, XCIII-XCVI). Su questo gruppo di sonetti rimando allo studio di Tufano (2007: 147-183).

6 I capitoli XIV, 3 e 4 delle Geneaologie di Boccaccio mettono a confronto la poesia con la filosofia e la giurisprudenza, mentre l'Invectiva contra medicum di Petrarca, per cui è fondamentale lo studio di Bausi (2008), costituisce un ottimo esempio di conflitto tra artes liberales e mechanicae.

7 Cfr. Dionisotti (55-88) e Gaeta (149-255). 
dal bisogno di appoggiarsi organicamente a una corte, il più delle volte estranea ai propri valori, oppure di intraprendere la carriera ecclesiastica. Se Dante propone una letteratura 'forte', agonistica, che incida e intervenga "in hac vita" come monito universale ("si enim ipse, et alii poterunt"), Petrarca e Boccaccio si rivolgono nelle opere latine a un pubblico di soli dotti $^{8}$. E se per Dante la gloria poetica è un bene funzionale al ritorno in patria e di durata storicamente determinata (vd. Paradiso, XXV, 1-9 e Purgatorio XI, 91-93), per gli altri due autori assume la valenza di fama eterna valida di per sé e da raggiungere individualmente.

Ciononostante, le prime risposte fornite da Mussato e Petrarca alla questione ci mostrano che il problema non era ancora colto nella sua globalità. Difatti, in estrema sintesi, Mussato nel Carmen XVIII rimane vincolato al modello convenzionale per cui la poesia ha una sorta di origine divina, poiché si basa su contenuti teologici e filosofici'; mentre l'autore del Canzoniere, nella famosa lettera al fratello Gherardo (Fam. X, 4), si limita a dimostrare i punti di contatto tra la poesia e la teologia, ribadendone il valore allegorico. Petrarca tenta sì di riscattare la poesia dall'inevitabile posizione di subalternità — cfr. X, 4, 1: "parum abest quin dicam theologiam poeticam esse de Deo" ("potrei persino dire che la teologia è la poesia che ha per oggetto Dio") — ma concede spesso restrizioni che ne intaccano l'autonomia (es. X, 4, 9): "non quod ideo carmen expetendum censeam; ne spernendum quidem" ("né dico che si debba tendere sempre alla poesia; dico che non la si deve disprezzare") ${ }^{10}$.

Simili atteggiamenti sono comprensibili se pensiamo alla particolare realtà sociale e personale vissuta dai due autori, che avevano certo avvertito la necessità di trovare una definizione precisa della poesia, ma senza provare la medesima urgenza nel ridelineare il proprio ruolo: non dobbiamo dimenticare che Mussato, seppur capofila del movimento preumanistico padovano, partecipa attivamente alla politica cittadina (podestà di Lendinara nel 1297, ambasciatore presso Bonifacio VIII ed Enrico VII, impegnato militarmente nel 1314 contro Verona); in aggiunta, scrive l'Ecerinis, che combina il rinnovato studio della classicità con l'impegno comunale: la tragedia rievoca il drammatico regno a Padova di Ezzelino III da Romano (1237-1259) e cele-

8 Per le citazioni si vedano Epistole, XIII, 78 e 51.

9 Come ha dimostrato Witt: "Lo scopo finale di questa difesa era sfumare la distinzione tra poesia e teologia, insistendo sulla continuità tra la poesia antica e la Bibbia" (137).

10 Curtius (239-253) ravvisa in queste posizioni una piena aderenza alle categorie medievali. 
bra contestualmente il regime comunale locale, all'epoca insidiato dalle mire espansionistiche di Cangrande della Scala, vera minaccia contingente contro cui si rivolge il messaggio parenetico del testo. Petrarca, invece, nonostante viva la condizione di intellettuale deraciné, riesce a trasformare il rapporto con i potenti in termini strumentali e vantaggiosi:

In un uomo come Petrarca, ben consapevole della propria forza e del proprio prestigio intellettuale, il fatto di non sentirsi organico al potere era ben lungi dal costituire un elemento di frustrazione: era invece la riprova e la celebrazione della propria intima indipendenza ${ }^{11}$.

Egli ricerca protezioni che garantiscano la produzione di opere dall'alto spessore civile e morale da sottrarre al "secol noioso" (Triumphus cupidinis, 17); e, così, il rifiuto di un rapporto locale stretto —ricordiamo la rinuncia alla cattedra dello Studium fiorentino del 1351 - permette di comportarsi quale cittadino del mondo, studioso libero e disinteressato (cfr. Epistola ad posteros), che offre la propria autorevole parola a politici, pontefici, regnanti italiani ed europei. Oltretutto l'organizzazione ancora approssimativa delle Signorie autorizza Petrarca a rapportarsi con più autonomia al foedus cortigiano ed evitare l'assunzione di impegni vincolanti: i continui trasferimenti - Avignone, Verona, Parma, Mantova, Padova, Treviso, Venezia, Milano sono la prova dell'attenta salvaguardia del proprio otium.

La situazione di Boccaccio è, al contrario, assai differente, giacché egli sperimenta una fase storica di maggiore provvisorietà, in cui la coabitazione petrarchesca tra il disimpegno letterario e la professione cortigiana si fa impraticabile: l'aspirazione a una sistemazione a Napoli o in Romagna è a lungo disattesa e l'insofferenza verso la gestione comunale fiorentina trova pronto sfogo in alcuni amari scritti. Secondo Gaeta (222), nella Consolatoria a Pino dé Rossi (1361-1362) ${ }^{12}$, il certaldese non solo dimostra di non amare il nuovo

11 Gaeta, 207-208. Sul tema è fondamentale Dotti.

12 Cfr. $\$$ 35-37: "Senza che, se alcuno luogo a spirito punto schivo fu noioso a vedere o ad abitarvi, la nostra città mi pare uno di quelli, se a coloro riguarderemo e a' loro costumi, nelle mani de' quali, per la sciocchezza o malvagità di coloro che avuto l'hanno a fare, le redine del governo della nostra repubblica date sono. [...] $\mathrm{E}$ acciò che io più di questi non conti (per ciò che non me ne maraviglio, pensando che non simili alle fortune piovano da Dio gli animi ne' mortali), eziandio a quali noi vogliamo più originali cittadini divegnendo, quelli o per avere d'insaziabile invidia gli animi occupati o di superbia intollerabile enfiati o d'ira non convenevole accesi, non l'avere pubblico ma il proprio procurando, hanno in miseria tirata e tirano in servitudine la città, la quale ora diciamo nostra e della quale, se modo non si muta, ancora ci dorrà essere chiamati. E 
regime presso cui prestava servizio, ma "man mano che gli anni passavano, diventa sempre più critico nei suoi riguardi, sempre più scettico nei confronti di ogni impegno politico, sempre più disincantato in materia di rapporti con il potere"; non a caso nel Trattatello in laude di Dante (\$ 64) arriva a criticare l'ardore e la passione politica profusa dal venerato concittadino, in quanto segno di asservimento che ostacola la poesia: "fermossi adunque Dante a volere seguire gli onori caduci e la vana pompa dei publici ofici"13.

Stabilite le divergenze all'internop dei tre panorami storici e biografici, sembra comprensibile che Boccaccio ricercasse tanto febbrilmente di legittimare la sua produzione ${ }^{14}$. Per di più, non solo lo scrittore difende ed esalta in molte opere il valore della letteratura, ma giunge anche a un livello di profondità inedito. La critica, infatti, si è soffermata da tempo sulle considerazioni esposte all'interno del XIV libro delle Genealogie, evidenziandone la portata innovativa ${ }^{15}$. Siccome gli elementi messi in campo da Boccaccio sono ormai risaputi, mi limito a un sunto cursorio: prima di tutto, per giustificare i contenuti delle scritture pagane, il certaldese tende a restringere il valore della retorica, concedendo all'inventio un suo inalienabile diritto ${ }^{16}$. La poesia, dunque, non si definisce a partire dalla tecnica,

oltre a ciò vi veggiamo (acciò ch'io taccia per meno vergogna di noi i ghiottoni, $\mathrm{i}$ puttanieri, i tavernieri e gli altri di simili lordure) disonesti uomini assai, i quali, quale con contenenza gravissima, quale con non dire mai parola e chi con l'andare grattando i piedi alle dipinture e molti collo anfanare e mostrarsi tenerissimi padri e protettori del comune bene (i quali tutti, ricercando, non si troverà sappiano annoverare quante dita abbiano nelle mani, come che del rubare, quando fatto loro vegna e del barattare sieno maestri sovrani), essendo buoni uomini riputati dagl'ingannati, al timone di sì gran legno, in tante tempeste faticato, sono posti”.

13 Altre estese critiche verso Firenze si possono rintracciare ai paragrafi 92-109. Nel citare l'opera, si farà riferimento alla prima redazione.

14 Riflessioni a riguardo sono rintracciabili in: Decameron (Proemio, Introduzione alla IV giornata e Conclusione); Trattatello (soprattutto $\$ 125-162$ ); Genealogie (XIV e XV); De mulieribus claris (XXVII); De Casibus (III, 6 e 14); Vita di Petrarca (IX e XXVI) ed Esposizioni (I, 3).

15 Branca ha osservato che nelle Genealogie la difesa della poesia "è meno cauta, meno retorica di quanto non lo sia nel Petrarca: è piena, totale, appassionata. [...] La poesia per la prima volta è definita in quanto poesia" (287). Tra gli studi più importanti segnalo quelli di Barberi; Canetti; Cazalé Bérard; Chines; Gilson; Hortis; Kriesel; Martellotti; Papio; Stefanelli; Tateo.

16 Genealogie XIV, 7, 1: "Poesis enim, quam negligentes abiciunt et ignari, est fervor quidam exquisite inveniendi atque dicendi, seu scribendi, quod inveneris. Qui, ex sinu dei procedens, paucis mentibus, ut arbitror, in creatione conceditur, ex quo, quoniam mirabilis sit, rarissimi semper fuere poete" "La poesia - che gli ignoranti e i 
ma possiede una pulchritudo, ossia una pienezza di senso tale da renderla esclusiva ${ }^{17}$. Essa si caratterizza, così, come una "succiplena facultas" $(6,9)$ dotata di un codice di rimandi e sovrasensi autonomi e dall'elevata matrice culturale. Poi Boccaccio decide di affrontare la tematica intorno all'equiparazione tra poesia e teologia (10); tuttavia qui non segue il comune procedimento medievale, anzi elimina, come osserva Tateo (op. cit., p. 75):

dal termine di teologia, quanto poteva esserci di più specificatamente religioso, di più veramente teologico, svuotando il termine del suo significato cristiano e storicamente significativo, per assumerlo nel più indeterminato significato di ricerca della verità più profonda del mondo.

Oltre a ciò Boccaccio confuta la formulazione dantesca di poesia come "fictio" e rifugge l'etimologia che, collegando "poio" a "poesis" $(7,4)$, ne faceva risaltare il carattere mendace. Il certaldese, quindi, propone la nuova definizione di "exquisita locutio" $(7,4)$, capace di mettere in secondo piano la portata di mera finzione dell'invenzione artistica e, allo stesso tempo, di esaltarne le qualità intrinseche. Ciò spinge Boccaccio ad arricchire l'espressione connotativa e parlare di esemplarità $(9,4:$ "Fabula est exemplaris seu demonstrativa sub figmento locutio, cuius amoto cortice, patet intentio fabulantis"; "La favola è un'espressione esemplificativa o dimostrativa sotto il velo della finzione; e, rimossane la corteccia, è manifesta l'intenzione di colui che favoleggia"): la poesia è una scientia superiore che riesce a far convivere le proprietà essenziali di utile e validità morale con quelle proprie di una "honesta fabula" (9, 1-7). Per mezzo di quest'ultima nozione, si trasmette l'idea che il poeta non imiti la natura e la realtà allo scopo di fissare alcuni paradigmi immutabili, bensì rappresenti infinite possibilità entro una vasta serie di combinazioni $(7,8)$ : "mera poesis est quidquid sub velamento componitur et exponitur exquisite" ("è pura poesia tutto ciò che componiamo sotto il velo dell'allegoria e ciò che accuratamente si espone"). Pertanto, viene superato il concetto tradizionale di allegoria: non è la verità che conta, quanto la composizione letteraria, che acquista un'alta condizione di autonomia e di significato ("integumenta" a 7, 8) nel momento in

i negligenti rifiutano - è un certo fervore di trovare pensieri eletti e di dire e descrivere ciò che si è trovato. Questo fervore, procedendo dal grembo divino, a pochi menti - come credo - è concesso nella creazione; e perciò, poiché è mirabile, i poeti furono sempre rarissimi”).

17 Genealogie XIV 6, 5: "Nonne vident ignari ipsum nominis huius, facultatis' scilicet, significatum semper aliqualem plenitudinem demonstrare?" ("Non vedono, gli ignoranti, che lo stesso significato di quel nome, cioè 'arte', dimostra sempre una certa pienezza?"). 
cui diventa espressione di un'eventuale verità. Si rompe pure l'inscindibile collegamento tra pulchrum e bonum, dacché lo scrittore, senza rinunciare a una sua moralità, non è costretto a farsi portatore del messaggio cristiano. Ciò consente al poeta di attingere dalla traditio classica momenti, figure, storie e personaggi che hanno ormai conseguito la piena legittimità letteraria. E, quindi, citando San Paolo (18, 10: "Scire malum, malum non esse sed operari"; "non è male conoscere il male, ma commetterlo"), viene provata, anche con l'avallo di una delle massime autorità cristiane, l'indipendenza e l'utilità della letteratura come fonte di conoscenza e crescita ${ }^{18}$. E l'appassionato appello ai lettori, collocato alla fine del dodicesimo capitolo (17), spiega che l'ambiguità della realtà richiede di partire da una base umana per afferrare le leggi dell'armonia universale:

Et ut iterum dixerim, volentibus intelligere et nexus ambiguos enodare legendum est, insistendum vigilandumque, atque interrogandum, et omni modo premende cerebri vires! Et si non una via potest quis pervenire, quo cupit, intret alteram, et, si obstent obices, arripiat aliam, donec, si valiture sint vires, lucidum illi appareat, quod primo videbatur obscurum ${ }^{19}$.

Ora, se gli studiosi hanno analizzato con acribia il libro XIV delle Genealogie, indicando le articolate argomentazioni a difesa ed emancipa-

18 Il santo viene evocato pure nelle Esposizioni (I, 1, “poeta fui”, $\$ 100-101$ ) per sottolineare la libertà non solo della letteratura, ma anche dell' atto di lettura stesso: "Appresso, se essi nol sanno, leggano negli Atti degli Apostoli e troveranno se Paolo, vaso d'elezione, studiò i versi poetici e quelli conobbe e seppe: essi troveranno lui non avere avuto in fastidio, disputando nello Ariopago contro la ostinazione degli Ateniesi, d'usare la testimonianza de' poeti; e in altra parte avere usato il testimonio di Menandro, comico poeta, quando disse: 'Corrumpunt bonos mores colloquia mala'. E similemente, se io bene mi ricordo, egli allega un verso di Epimenide poeta, il quale attissimamente si potrebbe dire contro a questi sprezatori de' poeti, quando dice: 'Cretenses semper mendaces, male bestie, ventres pigri'. E così colui il quale fu rapito insino al terzo cielo non estimò quello che questi, più santi di lui, vogliono, cioè esser peccato o abominevole cosa aver letti e apparati i versi de' poeti".

19 "E per ripetermi, a chi vuole capire e sciogliere i nodi dell'ambiguità, occorre leggere, star sopra ai testi, vegliare, interrogare e in ogni modo premere le forze del cervello. E se per una via non si possa pervenire dove si desidera, si entri per un'altra: e se si frappongono ostacoli, se ne prenda una terza, fino a tanto che, se basteranno le forze, appaia chiaro quello che prima sembrava oscuro". Chiosa Tateo: "La poesia si ricollega così alla storia civile dell'uomo, realizzandone la capacità, non in quanto egli sia capace di unirsi misticamente a Dio, $[\ldots] \mathrm{ma}$ in quanto egli si allontana [...] per realizzarsi quale uomo" (114). 
zione della poesia, sembra però utile ritornare sulla discussione circa il ruolo dei poeti e il rapporto con la committenza. Tali temi, indispensabili per capire a fondo il ragionamento e la metodologia di Boccaccio, sono talmente importanti da illuminare l'opera sotto una diversa prospettiva. Cercherò, dunque, di concentrare l'analisi sugli ultimi due libri delle Genealogie, richiamando però gli altri testi in cui l'autore medita sulle categorie menzionate; difatti egli non esegue mai un discorso sistematico ed esaustivo, ma tratteggia a più riprese spunti e suggestioni da ricollegare e allacciare insieme.

Già il proemio dell'opera è organizzato in modo da indirizzare il lettore con precisione: Boccaccio si rivolge al committente delle Geneaologie Ugo IV di Lusignano, re di Cipro e Gerusalemme ${ }^{20}$ - e al suo segretario, Donnino da Parma, sostenendo di esser pronto ad affrontare il lavoro richiesto, vale a dire esporre la "genealogiam deorum gentilium et heroum ex eis iuxta fictiones veterum descendentium" (I, 1, 1; "la genealogia degli dei pagani e degli eroi che da essi discendono"). Sottolineando la gravosità dell'impresa per la mole del materiale e la difficoltà nel raccoglierlo, lo scrittore esalta indirettamente il compito di cui è stato incaricato ${ }^{21}$. Pur tuttavia il nome di Ugo IV dovrebbe destare subito numerose perplessità, poiché il re, al momento della conclusione dell'opera (primi anni Settanta, ma iniziata a scrivere nel 1360, era deceduto nel 1359. Penso che, dietro questo particolare non secondario, possano emergere alcuni dati significativi: l'acuta osservazione di Zaccaria (1998: 1611), secondo cui Ugo IV occupi una posizione letteraria, è stata sin ora evasa. Ma credo giovi approfondire questa strada per motivi sia di logica interna al testo sia strutturali: Boccaccio confessa di non aver mai visto il sovrano (XV, 13, 4), che interpella sempre come vivo. Sarebbe risultata un'assurda celebrazione, però, ignorarne la morte senza mai fare accenno alla scomparsa. È difficile da sostenere, poi, che un meticoloso revisore e riscrittore come Boccaccio abbia omesso un particolare fondamentale all'interno di un'opera dalla

20 Per informazioni sul sovrano si vedano Grousset e Hortis (139-161).

21 Cfr. $\$$ 31: “Ceterum si cetera pepercissent, non eis, restauratore carentibus, pepercisset labile tempus, cui et taciti et adamantini sunt dentes, nedum libros, sed saxa conterentes durissima et ferrum ipsum, domans cetera" ("Ma anche se tutto il resto li avesse risparmiati, non li avrebbe risparmiati, privi com'erano di un restauratore, il tempo fugace che ha denti nascosti e duri come il diamante, che corrodono non solo i libri, ma anche i più duri sassi e perfino il ferro che doma tutte le altre cose"). E ciò raggiungerà il culmine nel proemio del libro XIV (3), in cui Boccaccio sostiene di essere riuscito nell'impresa "iuxta promissum" contro ogni previsione. 
grande rilevanza. Ed è altrettanto singolare ritenere che l'autore non sia mai stato informato del fatto; ciò dimostrerebbe soltanto l'inconsistenza del rapporto di committenza. Più semplicemente ipotizziamo che, se costui, ormai trapassato, è rappresentato come ancora vivo, venga caratterizzato a partire da una dimensione artistica: a ben guardare il sovrano assume un ruolo più marcato del semplice committente rarefatto e statico, in quanto viene sovente nominato (es. VII, 1; IX, 1; X, 1 e XIV, 20 e 21), ma mai in modo futile o banale; anzi l'accenno serve di solito a intrattenere un dialogo, a stabilire un contatto. A V, 22, 11, per esempio, l'autorità di Ugo IV è menzionata quasi per giustificare l'utilizzo di una preterizione, con cui si ricalca il motivo della recusatio dell'epos nella poesia augustea (cfr. Orazio, Odi I, 6; Virgilio, Georgiche, III, 1-48; Properzio, II, 1): "serenissime rex, si huius tam grandis fabule ad unguem sensum enucleare voluerimus, in ingens profecto volumen evaderet, et ideo cur Apollinis, et Endilichie filia dicatur Psyces, que eius sorores, et cur Cupidinis dicatur coniunx, cum paucis ex contingentibus dixisse satis sit" "'Serenissimo re, se volessimo enucleare con precisione il significato di questa lunga favola, ne dovrebbe uscire un gran libro; e perciò basti dire perché Psiche sia detta figlia di Apollo e di Entelechia, chi siano le sorelle, perché ella sia detta sposa di Cupido, insieme con altri pochi argomenti che la riguardano"); mentre nel libro XIV (8, 1) egli assolve il medesimo compito del Lucilio senecano: "si, mi rex, qua sub celi plaga, quibus seculis, cuius opere hec primo comparuerit in terris, exquiras, vix credo satis certum posse dari responsum" ("se tu, mio re, cercassi in qual parte del mondo, in che tempo, per opera di chi, primieramente la poesia sia comparsa in terra, credo che a stenti si possa dare una risposta abbastanza sicura")22. Come Seneca nelle Epistulae, anche qui il certaldese ha bisogno di un destinatario con cui parlare e confrontarsi. Il discorso intrattenuto, nondimeno, non è unilaterale, in quanto Boccaccio a volte anticipa i pensieri del committente e ne indirizza il ragionamento in funzione pedagogica (es. XIV, 20, 3): "advertis ne, prudentissime rex, in quem exitum tendat illecebris horum astutia? Sed, qualiscunque sit, veritate lucida

22 Cfr. ad esempio Epistuale, III, 23, 7: "Quod sit istud interrogas, aut unde subeat? Dicam" ("quale sia questo bene, tu chiedi, e da dove derivi? Te lo dirò"). I punti di contatto tra le Genealogie e il Seneca 'morale', uno tra gli auctorores prediletti del Medioevo, andrebbero indagati con attenzione. La critica ha sinora sottolineato i rapporti intertestuali con le opere erudite o le tragedie; mi sembra, invece, che i Dialoghi e le Epistulae assumano un valore più profondo. Si pensi soltanto alle violente critiche contro i pessimi filosofi (XIV, 3) o gli avvocati e intellettuali assetati di guadagni (XIV, 4 e 5), assemblate a partire da numerosi punti del De brevitate vitae (es. XII-XIV). 
confundenda est" ("avverti, prudentissimi re, verso qual fine tenda l'astuzia seduttrice di costoro? Ma qualunque essa sia, bisogna abbatterla con la limpida verità). L'equilibrio ottenuto tra la descrizione storica del personaggio e, di contro, tra la sua trasfigurazione, pare consentire allo scrittore di rendere a volte Ugo IV come un 'tu' preciso e in altri casi quale 'tu' generico, che simboleggia l'intera comunità di fruitori.

L'espediente di inserire un committente fittizio sembra, quindi, costituire l'ennesimo elogio delle potenzialità letterarie, tanto che l'excusatio non petita di XV, 13, dal titolo palese di "vero, non ficto regis mandato hoc opus compositum" ("quest'opera è stata composta per vero, non per finto comando di re"), celebrerebbe di riflesso tale realtà. Non è importante interrogarsi se il sovrano fosse fittizio già nelle prime fasi di stesura dell'opera, quanto constatarne l'inconsistenza finale come figura storica e precisare, di contro, il profilo retorico-letterario ${ }^{23}$. Impiegare una personalità famosa ed esotica come dedicatario non solo avvalora un'operazione assai ardua e sperimentale, ma potrebbe affermare la superiorità della poesia sul potere politico: con un espediente raffinato, Boccaccio dimostra che la letteratura è indispensabile per eternare la vita umana e, quindi, che la committenza ne dipende in subordine. Infatti i grandi personaggi storici hanno bisogno dei poeti per perpetuare il proprio ricordo; al contrario, la letteratura ha un margine d'azione più ampio, tanto che può prescindere dalla committenza e, nel caso di Boccaccio, addirittura inventarsela. Del resto lo scrittore bada a rendere inequivocabile il concetto, quando afferma che le Genealogie erano state richieste da un altro sovrano ${ }^{24}$. L'epigrafica glossa seguente non

23 Il capitolo tredicesimo del libro XV attesta gli incontri avuti con Donnino e Becchino Bellincioni, finalizzati a convincere Boccaccio a scrivere l'opera; la richiesta ufficiale sembra essere stata consegnata da Paolo Dagomari "sigillo Maiestatis". Tuttavia la nota inclinazione boccacciana alla falsificazione, per cui rimando a Bologna, ci suggerisce di accogliere con prudenza tali testimonianze.

$24 \mathrm{Cfr}$. XV, 13, 5: "Si opus meum insignire regio nomine cupiebam, prope erat, imo coram (nec verebor dicere) rex, qui, si credidisset obtinuisse, postquam ceperam, quod minime in animo erat, dum me convenit Domninus, viva voce rogasset, ut sue hoc opus ascriberem maiestati, esto minus intelligens princeps esset, existimans non michi suo nomine gloriam inferre, quin imo meis licteris eternum suis titulis decus addere" ("Se desideravo insignire la mia opera del nome reale, era vicino, anzi era alla mia presenza - e non temo di dirlo - un re che, se avesse creduto di poter ottenere - quando già avevo cominciato - ciò che non era mia intenzione di chiedere, quando mi incontrò Donnino, avrebbe chiesto a viva voce che dedicassi quest'opera alla sua Maestà, pur essendo principe meno intelligente; stimando che, non a me, il suo nome recasse gloria, anzi che con i miei scritti avrei aggiunto ai suoi titoli uno splendore eterno"). 
potrebbe essere più esplicita (XV, 13, 6): "nec mirum; suffragiis enim scriptorum stant insignia et nomina regum" ("né è da meravigliarsene: le insegne e i nomi dei re si fondano sull'aiuto degli scrittori")25. E di seguito Boccaccio riporta alcuni aneddoti storici significativi (6): Alessandro Magno, durante la campagna di Persia, portò con sé "multos scriptores" per descrivere le sue gesta e, giunto sulla tomba di Achille, confessò che la gloria dei sovrani discende dagli scrittori; Pompeo donò la cittadinanza romana al poeta Teofane di Mitilene "quasi nomen suum perenne facturum"; inoltre gli Scipioni, Catone, Mario, Cicerone e Metello furono benevoli verso alcuni scrittori "ut de se scriberent".

Tali ragionamenti devono essere ricondotti alle riflessioni di XV, 11, in cui Boccaccio si discolpa dall'accusa di narrare vicende empie e sconvenienti intorno ad alcuni regnanti. Per prima cosa egli suggerisce che un buono scrittore non debba peccare di tracotanza $(11,2)$ : "agit enim temerarie, qui in nimium terminos excedit audacie; sic memini in libro Ethycorum Aristotilem arbitrari. Hos ego excessisse non reor; audere quidem, quod ab omni necessitate concessum est, non est temerarie agere" ("sconsideratamente agisce chi valica di troppo i confini dell'audacia; e così, ricordo, opina Aristotele nell'Eticha. Io non credo di aver superato questi limiti: poiché osare ciò che è concesso da ogni opportunità non significa agire sconsideratamente"). E poi sposta l'attenzione sui comportamenti dei sovrani, innalzando, per l'ennesima volta, l'autosufficienza dell'arte: "nemini prohibitum legi, seu honesta sint, seu etiam minus honesta, regum gesta describere; erat tamen regibus satius sic egisse, ut nil de eis referri posset preter honestum" ("ho letto che a nessuno è proibito di descrivere le gesta dei re, sia oneste che disoneste; sarebbe meglio tuttavia che i re si comportassero in modo che nulla, se non onesto, di essi si potesse riferire").

All'interno del tredicesimo capitolo, lo scrittore lascia un'altra significativa testimonianza, in quanto afferma che la dedica a un re rappresenta un unicum all'interno della sua produzione (XV, 13, 7)26. Tuttavia Boccaccio vi ricorre in altre occasioni, scegliendo amici e conoscenti e legando sempre l'atto di omaggio alla riflessione letteraria: la Comedia delle ninfe fiorentine (1341-1342) viene dedicata a Niccolò di Bartolo del

25 Più sotto viene ribadito (XV, 13, 8): "Quid multa? Non equidem magnorum ducum nomina claros scriptores faciunt, imo potius ipsi reges scriptorum opere cognoscuntur a posteris" ("a che dir molto? Non davvero i nomi di grandi condottieri rendono illustri gli scrittori, anzi perfino i re sono conosciuti dai posteri per opera degli scrittori”).

26 Limportanza di analizzare le modalità e le implicazioni delle dediche all'interno dell'opera letteraria è sottolineato dai lavori di Genette, Paoli e Terzoli. 
Buono; il De mulieribus claris (1361-1362) - all'interno del quale (XXVII) l'autore si ricava uno spazio per trattare il tema della nascita delle lettere e del valore della scrittura - ad Andreola Acciaiuoli; mentre il De casibus virorum illustrium (1356-1373), anch'esso non privo di digressioni teoriche (es. III, 14 e VI, 13), a Mainardo de' Cavalcanti. Nello specifico, l'ultimo capitolo della Comedia (L), che funge da commiato verso i lettori, viene impiegato per salutare il dedicatario: Boccaccio connota con precisione Niccolò - cfr. $\$ 3$ : "tu, o solo amico, e di vera amistà veracissimo essemplo" - al quale chiede di accogliere l'opera appena conclusa: "prendi questa rosa, tra le spine della mia avversità nata, la quale a forza fuori de' rigidi pruni tirò la fiorentina bellezza, me nell'infimo stante delle tristizie, dando sé a me con corto diletto a disegnarsi”. Il tono è mesto e si ricollega agli ultimi versi del capitolo ternario antecedente (cfr. vv. 94-100, cui si contrappongono i gai endecasillabi 1-21 e 52-81):

Io mi tornai, dolendo de' miei mali, al luogo usato; e attendendo peggio per la sua fine, ho già pennute l'ali al volare alla morte, la qual cheggio la notte e 'l dì per men doglia sentire, però che bene altro fin non veggio esser serbato al mio lungo martire.

Il dolore per la fine dell'esperienza poetica e il ritorno alla realtà quotidiana gettano l'autore, sradicato dal suo ambiente naturale, nello sconforto più cupo. Ciò nonostante Boccaccio recupera parte dell'entusiasmo svanito, esplicitando l'alto compito dell'opera e immettendola all'interno di una tradizione illustre ${ }^{27}$.

Nel De mulieribus il certaldese, opponendo la nobiltà della dedicataria al "vulgus iners", confessa di aver cercato a lungo la donna "dignior" cui porgere il testo (Giovanna, regina di Gerusalemme e di Sicilia e "multae aliae"), ma di essere giunto, alfine, a una scelta saggia, perché Andreola rappresenta l'onestà, il decoro e l'eleganza di un'epoca ormai perduta di cui si vorrebbe recuperare almeno sulla memoria ${ }^{28}$.

27 Vd. il seguente periodo (\$ 4): "E questa [scil. rosa] non altrimenti ricevi che da Virgilio il buono Augusto o Erennio da Cicerone, o come da Orazio il suo Mecena, prendevano i cari versi, nella memoria riducendoti l'autorità di Catone dicente: Quando il povero amico un picciolo dono ti presenta, piacevolmente il ricevi”.

28 Cfr. $\$$ 6: “Cum tempestate nostra multis atque splendidis facinoribus agentibus clarissimum vetustatis specimen sis" ("tu sei nell'età nostra, per molte splendide azioni, fulgido esempio di ciò che fu il mondo antico"). 
Ma è l'estesa dedica del De casibus a orientarci con chiarezza; anche in questo frangente Boccaccio confessa di aver nutrito alcuni dubbi $(\$ 2)$ : "non enim satis mecum conveniebam cui nam primo illud mictere vellem" ("non sapevo decidermi cui per primo dedicarlo"). La riflessione si fa assai ampia e, assumendo un carattere programmatico, merita di essere seguita nei suoi snodi fondamentali: l'autore spiega l'indecisione illustrando i meccanismi che spingono gli scrittori a dedicare le proprie opere. Costoro cercano di nobilitarle, rendendole "diutiores" e splendide (\$3); e, per tale motivo, si mettono alla ricerca di personaggi famosi. Ma la situazione contingente ha fatto sì che i pontefici "moderni" (\$ 6) siano indegni di un onore siffatto, poiché dediti ai denari, alle guerre, al potere "adversus veritatis Verbum"; l'imperatore, "hodiernus cesar" (\$7), antepone il vino e il lusso alla memoria dei gloriosi predecessori; i sovrani locali più rappresentativi ( $\$ 8-9)$, invece, vengono dipinti come ignoranti, "semibarbari et efferati homines... seri... molles et effermminati". Ma, quando l'avvilimento sembra aver vinto il poeta, ecco che Boccaccio predilige un "amicus". Mainardo - sebbene "phylosophicis eruditus non sit", al $\$ 15$ - personifica un ottimo interlocutore perché è "amantissimus studiorum...probatorum hominum precipuus cultor...solertissimus indagator" e, oltretutto, è portavoce, come Andreola, di quella classe nobile e aristocratica, da sempre ammirata dallo scrittore ${ }^{29}$.

Il raffronto tra le dediche permette di avanzare alcune considerazioni complessive: l'esibito procedimento attraverso il quale il certaldese mostra di aver scelto il dedicatario non va a sminuire chi gode dell'omaggio, ma semplicemente esplicita la contrapposizione tra il vulgus e i lettori - e mecenati-protettori - ideali. La cesura, difatti, non viene posta a livello sociale - e in ciò vengono mantenuti i convincimenti espressi nel Decameron - bensì riguarda l'aspetto umano e culturale delle singole persone $^{30}$. Gli amici Niccolò, politico fiorentino, mercante e futuro opposito-

29 Per notizie biografiche dettagliate sui tre dedicatari rimando a Klein; Miglio e Tanfani.

30 Gaeta ha posto l'accento sul "rifiuto [scil. boccacciano] del mondo contemporaneo caratterizzato dallo sviluppo di un'alta civiltà urbana dal quale l'intellettuale doveva ritrarsi per prendere le distanze da un 'volgo' che finiva per comprendere papi, re, principi, nobiltà, ricchi, arricchiti e popolo" (225). Le affermazioni dello studioso possono essere avvalorate da un episodio non marginale, in quanto scevro da dilatazione letteraria: in una nota del Par. lat. 4939, contenente il Compendium del vescovo Paolino da Pozzuoli, Boccaccio glossa così il testo, bollando l'elogio acritico verso Giovanni XXII: "Iste Venetus adulator nicil dicit de tyrampnide gesta per papam istum, de trucidatione christianorum facta suo 
re nel 1360 della politica comunale, Andreola e Mainardo, personaggi di spicco del regno di Napoli, tanto rimpianto dallo scrittore, incarnano la possibilità concreta per chi fa letteratura di scegliere non solo la materia da trattare, ma pure a chi destinarla e con quali scopi e forme.

Non pare casuale, dunque, che la consapevolezza nei mezzi del proprio labor poetico pervada le Genealogie: a XV, 10, per riportare solo un brano, si discute, con rigore e approccio quasi lucreziano, sulla necessità che ognu ${ }^{30}$ Gaeta ha posto l'accento sul "rifiuto [scil. boccacciano] del mondo contemporaneo caratterizzato dallo sviluppo di un'alta civiltà urbana dal quale l'intellettuale doveva ritrarsi per prendere le distanze da un 'volgo' che finiva per comprendere papi, re, principi, nobiltà, ricchi, arricchiti e popolo" (225). Le affermazioni dello studioso possono essere avvalorate da un episodio non marginale, in quanto scevro da dilatazione letteraria: in una nota del Par. lat. 4939, contenente il Compendium del vescovo Paolino da Pozzuoli, Boccaccio glossa così il testo, bollando l'elogio acritico verso Giovanni XXII: "Iste Venetus adulator nicil dicit de tyrampnide gesta per papam istum, de trucidatione christianorum facta suo iussu, de partialitate animosa eiusdem et de quam pluribus aliis dyabolicis gestis eiusdem" ("questo adulatore veneto non dice nulla sui comportamenti dispotici zno segua, secondo natura, le inclinazioni personali31. Alla libertà della poesia corrisponde quella del poeta di scegliere come professare la propria disciplina. Limpostazione argomentativa di carattere generale viene avvalorata dalle vicende biografiche del poeta (10, 6-7): "verum ad quoscunque actus natura produxerit alios, me quidem experientia teste ad poeticas meditationes dispositum ex utero matris eduxit et meo iudicio in hoc natus sum" ("ma poi, a qualsiasi azione abbia generato altri, io sono stato da essa disposto e ne è

iussu, de partialitate animosa eiusdem et de quam pluribus aliis dyabolicis gestis eiusdem" ("questo adulatore veneto non dice nulla sui comportamenti dispotici commessi da questo papa, sulla trucidazione di cristiani partita dal suo ordine, sulla faziosità feroce di questo stesso personaggio e sui suoi moltissimi altri gesti diabolici"; la traduzione è mia). Paolino, attaccato in altri punti per i metodi filologici anacronistici, personifica qui la figura dell'intellettuale asservito al potere, che non riesce a svolgere la propria attività con la giusta autonomia. Per ulteriori informazioni rinvio a Costantini.

$31 \mathrm{Vd}$. XV, 10, 5: "Uti incommodum humani corporis inter se differentia qualitate et officio membra a natura rerum apposita sunt, ut ex hac diversitate consistat, uti melodia ex diversitate tonorum, sic et, ut humanum genus perseveret, necesse fuit ad studia inter se differentia gigneremur" ("come a vantaggio del corpo umano sono state disposte dalla natura membra fra sé diverse per qualità e compiti, in modo che esso possa consistere proprio in quelle diversità, come anche la melodia deriva dalle differenze di toni, così pure fu necessario, per la conservazione del genere umano, che fossimo generati con inclinazioni fra loro differenti”). 
testimone l'esperienza fin dal grembo della madre alle meditazioni poetiche e, a mio giudizio, sono nato a questo"). Con passione e una vena di malinconia Boccaccio rievoca i contrasti con il padre - che lo voleva mercante "dives exinde futurus" - e i primi entusiasmi per la poesia (10, 8): "et ecce, ipsa impellente natura, fingendi desiderium affuit" ("quand'ecco, sotto la spinta della natura, si accese il desiderio di comporre"). Si impongono, infine, nella memoria le proterve resistenze del genitore e il rammarico di essere giunto troppo tardi alla letteratura:

Nec dubito, dum etas in hoc aptior erat, si equo genitor tulisset animo, quin inter celebres poetas unus evasissem, verum dum in lucrosas artes primo, inde in lucrosam facultatem ingenium flectere conatur meum, factum est, ut nec negociator sim, nec evaderem canonista, et perderem poetam esse conspicuum ${ }^{32}$.

Grazie alla sublimazione delle potenzialità poetiche si manifesta l'opportunità di optare per un pubblico determinato e una committenza prestabilita: ciò risulta palmare a XIV, 1 , in cui Boccaccio, riprendendo il fitto dialogo con Ugo IV, declina alcune avvertenze di lettura, che assumono un valore programmatico. Il certaldese consiglia al sovrano di accogliere l'opera "benigne", di esaminarla per sommi capi, valutando poi se il compito affidato sia stato eseguito. L'elogio indiretto all'autore medesimo è abbastanza esplicito e viene accresciuto sul finale da un attestato di falsa modestia: "miraberis quod in tam protensum volumen postulatum tue claritatis evaserit, quantumcunque ob librorum penuriam multis in locis non satis integrum putem" ("ti meraviglierai che la richiesta della tua maestà abbia avuto esiti di così esteso volume, benché, per mancanza di libri, credo che in molte parti l'opera non sia abbastanza completa"). L'illustre lettore deve essere in grado, per giungere a una piena comprensione del testo, di afferrarne il "sensus" nascosto "sub rudi cortice". In questo modo il re giungerà a un sicuro appagamento, "mirabundus" $(1,2)$, rallegrandosi per aver assegnato il compito allo scrittore; questi si erge a massimo rappresentante dell'intera categoria, dotata di potenzialità preternaturali: "teque ipsum modesta quadam delectatione laudabis, quod iam dudum de poetis vera arbitratus sis, eos

32 "Non dubito però che, mentre l'età era allo scopo più adatta, se il padre lo avesse serenamente concesso, sarei riuscito qualcuno fra i poeti celebri; e invece, mentre si tentava di piegare il mio ingegno, prima ad arti lucrose e poi ad una disciplina da guadagno, è accaduto che io non sia né un mercante, né un canonista; e che perdessi l'occasione di essere un poeta ragguardevole". La questione della scelta poetica viene affrontata anche nel De casibus (III, 14, 2-11), sotto lo schema della Priamel di origine oraziana (Carmina, I, 1). 
scilicet non fabulosos simpliciter fuisse homines, ut invidi quidam volunt, sed eruditissimos quidem atque divino quodam animo et artificio preditos" ("e con certo moderato diletto ti compiacerai per aver già prima dato un giudizio vero sui poeti; che essi, cioè, non sono semplicemente dei cantafavole - come alcuni invidiosi vorrebbero - ma dottissimi e dotati di anima e di arte quasi divina"). Pertanto, i poeti non sono soltanto dei funamboli della parola, pronti a inventare storie ornate da velami retorici, anzi, servendosi della loro arte e fantasia, riescono a oltrepassare i limiti umani e giungere alle fonti della conoscenza. Ma la capacità di accedere al sapere non deve rimanere appannaggio di pochi eletti, bensì circolare quanto più tra i dotti $(1,4)$ : "cum iam visum illud in amicorum manus visendum tradideris, et tua licentia prodibit in medium" ("quando poi avrai vista l'opera e l'avrai consegnata nelle mani degli amici, perché sia esaminata, e col tuo permesso sarà pubblicata").

Esaminiamo, allora, quale tipo di lettore Boccaccio ricerchi per le Genealogie e, più in generale, per le opere latine della sua ultima fase creativa. A XV, 12 egli sostiene di aver scritto assai meno rispetto a quanto le fonti consultate avrebbero potuto permettere; la scelta è motivata dalla volontà di non spaventare il pubblico in un atto di lettura altrimenti scoraggiante. Tuttavia l'attenzione dello scrittore per i propri lettori viene bilanciata da una precisa selezione $(12,2)$ : "non enim puero aut inerti vulgo scribimus, quin imo, ut alias dictum est, doctissimo regi et provectis hominibus, si aliquando ex manibus tuis, serenissime princeps, ad alios venturum hoc opus est" ("non scrivo infatti per i ragazzi o per il volgo ignorante; al contrario - come altre volte ho detto - per un re dottissimo e per uomini esperti: se dalle tue mani, serenissimo principe, una volta quest'opera potrà giungere ad altri”). Quindi l'autore esclude una platea immatura - non ancora pronta a cogliere le elevate proposte recondite nel testo - e il volgo, nominato "iners" proprio come nella dedica del De mulieribus. Boccaccio, pertanto, non opera una valutazione classista, ma adotta un concetto estensivo del volgo, includendovi anche le fasce della popolazione più elevata, ma respinte, per motivi etici, politici o culturali, dalla sua attenzione. Del resto il pubblico elettivo viene indicato con un passaggio icastico e incisivo: Boccaccio è disposto a interloquire solo con i sovrani più raffinati e con gli uomini dalla sensibilità più avanzata. E nel libro XIV non pare casuale che la definizione del pubblico avvenga secondo l'opposizione con altre figure di intellettuali, incapaci di foggiare una classe dirigente all'altezza. Al capitolo secondo Boccaccio attacca gli ignari, rei di giudicare senza alcuna competenza su "omnia" ed elevare la mediocrità a metro di giudizio veloce e condiviso, più efficace delle "eruditorum hominum vigilias, meditationes et studia, honestosque labores et mode- 
stiam". Costoro vengono invitati a lasciar stare i "sapientes viri", dal momento che non esiste nulla di più sconveniente di un uomo "ignarus" e "indoctus" $(2,5)$. Nel capitolo susseguente vengono presi di mira coloro che desiderano apparire saggi, ascoltati con fedeltà dal "popellus ignarus". Questi assecondano gli istinti più bassi del proprio uditorio, denigrando le letture filosofiche fondamentali (es. Aristotele, Cicerone, Euclide) e disprezzando i poeti solamente al fine di intercettare gli applausi e le risa. Ma, come viene detto con tono senecano $(3,6)$ : "ignari sunt, et luce veritatis carentes, sensualitate duce se trahi permittunt" 33 .

A XIV, 4, invece, viene condotta la critica più appassionata "in iuris peritos": nel vasto capitolo la fisionomia dei "clarissimi legum preceptores" si allaccia saldamente con quella del popolo che li ascolta. Gli uomini di legge, a differenza di altri, non disprezzano la poesia e la cultura, ma le giudicano superficiali e inutili, poiché "post longos labores nulle consequitur opes" $(4,3)$. Pertanto, se le ricchezze costituiscono l'unico mezzo tangibile per valutare la dignità e il peso sociale di una professione, "nullius pretii eorum [scil. poetarum] extimanda facultas est”. Questo parere così parziale riesce comunque a penetrare negli animi degli ascoltatori meno avvertiti e a creare una communis opinio molto pericolosa, tanto che, entrando nel caso personale, "post multa dicturos opus scilicet pulchrum, sed supervaca-

33 Considerazioni affini sono presenti pure in XIV, 5; si leggano, nello specifico, i $₫$ 7-8: "His autem, decipulis captivatis insipientum iudiciis, civitates ambire, secularibus se miscere negociis, consilia prestare, connubia tractare, comesationibus interesse, testantium dictare tabulas, testamentorum executiones assummere, et multa minus phylosophos decentia agere presumptuose incipiunt atque prosequuntur. Ex quibus fit, ut in fumosam vulgi famam quandoque deveniant, ex qua tanta tumiditate turgescunt, ut incedentes cupiant a vulgo digito monstrari, et longe magis audire, quia pregrandes magistri sint, videre, quod eis assurgant presidentes in triviis, eos Rabi vocitent, salutent, invitent, preponant, atque preficiant" ("dopo essersi assicurati con queste trappole il giudizio favorevole degli ignoranti, cominciano, e continuano, ad andare in giro per la città, a mescolarsi agli affari profani , a offrire consigli, a trattar matrimoni, a partecipare a conviti, a dettare note di testamenti ad assumerne l'esecuzione e a comportarsi presuntuosamente, in molti casi in modo sconveniente a filosofi. Da ciò deriva che a volte giungano alla fumosa stima del volgo, per la quale si gonfiano al punto da desiderare di esser segnati a dito dal popolo stesso quando camminano; e più ancora di sentir dire che sono grandissimi maestri e di vedere che i nobili si alzano nei trivi al loro passaggio e li chiamano 'maestri' e li salutano e li invitano e li fanno passare avanti e si mettono al loro seguito"). La felice espressione "digito monstrari" è cavata da Persio (I, 28), altro autore presente nelle Genealogie (es. XIV, 15, 12; XIV, 18, 17 e XIV, 19, 14) e il cui peso intertestuale, in funzione dissacratoria, andrebbe scandagliato con cura. 
neum, et inutilem sudorem meum, eo quod minime tendat, quo ceterorum mortalium labores intendunt" (4, 4; "diranno, dopo molte parole, che l'opera è bella, ma vana ed inutile la mia fatica, perché non tende dove mirano gli sforzi degli altri uomini"). L'obiezione di Boccaccio non si fa certo attendere e pare servire, oltretutto, a definire il ruolo sociale dei poeti: prima di tutto divide le arti speculative, il cui fine consiste nello spingere "in desiderium eterni nominis" (4, 9), da quelle meccaniche, che puntano al guadagno. Poi sottolinea i vantaggi delle lettere, stabili e immortali, rispetto alla caducità delle leggi, sempre cangianti, per avvalerci di due termini propri della linguistica, sia diatopicamente sia diacronicamente. Segue poi una breve rassegna di scrittori celebri, che traccia una sorta di canone (4, 15-17): Omero, Ennio e Virgilio mantennero uno stile di vita dimesso; ciò nonostante, la loro reale ricchezza viene tramandata dalle opere e, non a caso, da alcuni importanti riconoscimenti da parte del potere politico. Difatti, quando Alessandro Magno vinse Dario, serbò una cassetta d'oro "pretiosissima", non ai propri gioielli, frutto del bottino di guerra, bensì "Homeri voluminibus"; il corpo di Ennio venne inumato nel sepolcro degli Scipioni, mentre l'Eneide è stata conservata grazie al pronto intervento di Ottaviano, che non rispettò la volontà di Virgilio di bruciare il capolavoro. La povertà, dunque, diviene una conditio sine qua non dell'attività poetica, perché è indispensabile al conseguimento della tranquillità d'animo, dell'ozio e della libertà dai condizionamenti esterni e dai turpi affanni $(4,21)$. La chiusura del brano, infine, ben sintetizza i due poli antitetici $(4,28)$ : Boccaccio ci presenta gli uomini di legge, mescolati alla folla nelle piazze straripanti mentre ricercano applausi vani e i poeti, all'opposto, quando meditano in solitudine esaltando le imprese degli uomini illustri.

Abbiamo visto come lo scrittore tuteli la propria professione, incalzata, da una parte, da una classe intellettuale variamente intesa (filosofi, giuristi, notai, accademici, insegnanti, teologi) sempre più competitiva e interdipendente e, dall'altra, dal potere politico con cui fatalmente cooperare. Tramite il tema della solitudine, però, egli sembra ribadire un'ulteriore specificità, e affermare un primato: a XIV, 11 i poeti vengono difesi dall'accusa di vivere in luoghi isolati perché rozzi o selvaggi. Per smentire tale assunto Boccaccio afferma che i poeti, poiché sono portati a convivere con re e nobili, sono i più adatti a relazionarsi con gli altri uomini. Ma questa asserzione - seguita dai consueti numerosi esempi, classici e italiani (Euripide, Ennio, Virgilio, Dante e Petrarca) - viene precisata sottolineando la libertà di scelta ("dum libuit" a 11,2) nel partecipare alla vita politica. La citazione di Orazio a 11, 5, che scrive a Floro opponendo gli strepiti diuturni della città alla pacifica vita di campagna, serve a presentare le due parti in conflitto: gli scrittori aspirano, attraverso la serenità, a un ambien- 
te in cui ottenere la "famosa gloria et per secula duratura" (11, 10), coloro che li attaccano, viceversa, bramano il potere e le ricchezze ${ }^{34}$.

A XIV, 19 viene ribadito il concetto, procurando ulteriori e più estesi esempi che, come i precedenti, ricoprono una posizione fondamentale nell'insieme delle tecniche argomentative boccacciane, giacché lo scrittore intende fornire ai contemporanei, partendo dai grandi maestri cui ispirarsi, alcune prove concrete di comportamento, che prescindono da letture religiose o allegoriche. Sembra nascere, anche grazie a questo atteggiamento, un modo nuovo di rapportarsi al mondo antico: la cesura con la classicità si avverte nella sua irrimediabile profondità, tanto da spronare, come fanno Boccaccio e Petrarca nelle loro opere erudite latine, al recupero, alla raccolta e alla riscoperta dell'immenso materiale documentario antico. Difatti gli aneddoti biografici di Omero, Virgilio e Petrarca, seguono una precisa direzione (11,3-9). L'autore dell'Iliade e dell'Odissea viene presentato con toni lirici e mitologici come colui che, dopo aver peregrinato per il mondo intero, si diede alla scrittura, cieco e indigente, nella desolata Arcadia. Questa vita isolata non lo distolse dalla sua arte e, in seguito, non ne impedì i riconoscimenti delle istituzioni panelleniche: "hunc insuper in suum civem plures Grecorum egregie civitates, eo etiam mortuo et paupere, voluere, et de hoc inter se movere litigium" ("inoltre molte illustri città greche vollero che Omero, anche dopo morto in povertà, fosse loro concittadino; e su ciò si mossero a contesa”). L'esistenza di Virgilio - come già visto, secondo un espediente accumulativo e didascalico frequente negli ultimi due libri delle Genealogie — termina con il salvataggio dell'Eneide da parte dell'imperatore e, qui viene aggiunto, con la sepoltura tra Posillipo e Pozzuoli, "haud procul ab electa solitudine". L'esperienza di un contemporaneo quale Petrarca, invece, serve a dimostrare che anche nel "nostrum evum" è possibile e fruttuoso seguire gli insegnamenti degli antichi: "nonne, spreta Babilone occidentali atque pontificis maximi benivolentia, quam omnes fere Christiani summopere cupiunt et procurant, et pilleatorum orbis cardinum aliorumque principum, in Vallem Clausam abiit, insignem Gallie solitudine locum [...]?”35.

34 I poeti, infatti (11,9): "Horrent fasces nedum exposcere, sed optare, aulas ambire regum, aut procerum quorumcunque assentatores fieri, auro pontificum infulas aucupari, ut ventri et inerti ocio latius indulgere queant" ("sdegnano non solo di chiedere, ma di desiderare il potere; di aggirarsi per le regge e di diventare adulatori di qualunque potente; di dar la caccia con l'oro alle insegne sacerdotali, per poter indulgere più largamente al ventre e all'ozio vile").

35 "Non forse, dopo aver disprezzata la Babilonia occidentale e trascurata la benevolenza del sommo pontefice, che quasi tutti i cristiani molto desiderano procurarsi, e di molti cardinale e di altri principi, andò in Valchiusa, famosa località solitaria della Gallia [...]?”. Anche nella Vita di Petrarca, che Villani colloca tra 
Resta da vedere, a quale scopo siano chiamati i poeti e come si esplichi il senso della loro attività. La riflessione più propriamente astratta viene affidata al De mulieribus e al De casibus, mentre le Genealogie coprono il lato più pratico e contingente della questione. Nel De mulieribus (XXVII) il certaldese - disquisendo intorno a Carmenta, la mitica inventrice dell'alfabeto latino - evidenzia l'aspetto eternatore della poesia (\$ 13-14). Il potere della scrittura - che mantiene la memoria dei grandi eventi come di quelli umili, permette di comunicare tra gli uomini e, attraverso la fantasia e l'intelligenza, rappresenta mondi sconosciuti sino a descrivere le divinità — viene sondato ripercorrendo tali importanti conquiste civili:

Quibus delinita, facultatum omnium infinita splendent volumina, hominum gesta Deique magnalia perpetua servantur memoria ut, que vidisse nequivimus ipsi, eis opitulantibus, cognoscamus. His vota nostra transmictimus et aliena cum fide suscipimus, his amicitias in longinquo iungimus et mutuis responsionibus conservamus. He Deum - prout fieri potest - nobis describunt; he celum terrasque et maria et animantia cuncta designant; nec est quod queras possibile quod ab his vigilans non possis percipere; harum breviter opere quicquid amplitudine mentis complecti atque teneri non potest, fidissime commendatur custodie. Que tamen, etsi aliis ex his non nulla contingant, nil tamen nostris commendabile aufertur ${ }^{36}$.

Al paragrafo 16, si lega la riflessione generale precedente con una considerazione sulla fortuna degli scrittori italiani, superiori ai colleghi europei - "rapacitas germana...gallicus furor...astutia anglica... hispana ferocitas... alicuius alterius nationis inculta barbaries vel insultus" — perché naturali detentori del patrimonio letterario classico.

il 1344 e il 1350 (20-30), la tematica della solitudine acquista una valenza strutturale $(\$ 9)$, legandosi al rapporto con i potenti $(\$ 12)$; esso non è mai letto in chiave di compromesso, anzi di amicizia e mutua cooperazione $(\$ 28)$.

36 "Risplendono infiniti volumi di tutte le discipline, scritti in caratteri latini; e per essi si serba perpetua memoria delle grandi imprese divine ed umane; cosicché col loro aiuto conosciamo quelle cose che noi non potremmo vedere. Per essi trasmettiamo le nostre suppliche e accogliamo con sincerità quelle di altri; per essi stringiamo lunghe amicizie e le conserviamo con reciproca corrispondenza. Essi, per quanto è possibile, ci descrivono Dio; essi indicano il cielo, i mari e tutti gli esseri animati; e non c'è cosa, tra quante si possa indagare, che per essi, con cura assidua, non possa essere appresa. In breve: quello che non può essere abbracciato e posseduto dalla nostra mente, si affida, per mezzo di loro, a sicurissima custodia; e tuttavia, anche se alcuni di questi meriti toccano ad altri alfabeti, ciò non significa che l'espressione di alcunché di commendevole possa essere sottratto al nostro". 
Nel De casibus, invece, viene stabilito un nesso tradizionale tra la bontà del poeta e quella dell'opera (III, 14, 13): "et si optimus homo sit, poesis optima apparebit". Questo passaggio si dà come obbligato, viste le considerazioni a VI, 13 che attribuiscono alla letteratura un compito assai impegnativo e ambizioso: la parola rappresenta la caratteristica distintiva dell'essere umano sugli altri animali $(\$ 3)$. Essa non permette solo di comunicare, ma, grazie all'arte, si trasforma nella modalità di espressione più nobile ed elevata. Come sintetizza l'autore, "non enim semper cibum servis poscituri sumus aut de ruralibus cum villico locuturi" (\$ 7); ecco che, allora, la letteratura consente all'uomo di accedere al complesso universo che lo circonda. Oltretutto viene indicata come necessaria pure da un punto di vista pratico per convincere le persone, consolarle, allietarle, esaltarle, calmarle e perfezionarle (\$10-15).

Le Genealogie procedono su un piano divergente; lo scrittore, date per scontate le finalità generali della letteratura, preferisce indicare i mezzi e gli obiettivi da considerare in relazione alla situazione politica e sociale presente. Come già analizzato, tale messaggio viene trasmesso a partire dalla presentazione di alcune carriere letterarie famose. A XV, 6 Boccaccio raffigura diversi intellettuali, accumunati in modo esplicito dalla particolare funzione pubblica e civile svolta; l'autore chiama in causa otto "insignes viri" moderni per difendere, ancora una volta, lo statuto poetico. Questa folta rassegna viene anticipata da una considerazione programmatica $(6,2)$ :

Ego autem huius sententie sum, nunquam in evum duraturos hos, quorum novitas approbata non sit, cum ab eorum novitate necesse sit exordium approbationis sumendum, et sic eos, quos ego novos invoco, cum vivos noverim aut noscam, meritis eorum agentibus, egregios esse viros atque probandos, ausus sum in testimonium evocare ${ }^{37}$.

Con una consapevolezza acuta dei cambiamenti in corso, Boccaccio sembra avvertire il richiamo di una imminente svolta culturale. La parola "novitas" - che riecheggia insistentemente tre volte con poliptoto e una con falsa etimologia per mezzo dell'accostamento allitterante con il congiuntivo perfetto di noscere - è impegnativa per la Weltanschauung classica e medievale e potrebbe testimoniare un vero spartiacque concettuale. La presa di coscienza della fine di un'epoca viene sottolineata con forza da queste pagine, che ci indicano, inoltre, le basi su cui far crescere le future generazioni. Se

37 "Io, per parte mia, sono del parere che non mai dureranno nel tempo quegli autori dei quali non sia approvata la novità; mentre della loro novità è necessario prendere il principio dell'approvazione; e così quelli che chiamano nuovi, avendoli conosciuti vivi e sapendo che per i loro meriti sono uomini egregi e degni di approvazione, ho osato chiamarli a testimoni”. 
Petrarca reagisce per sua ammissione ai mali presenti da laudator temporis acti (Sen. X, 2, 1), ponendo sé stesso come argine alla decadenza culturale e morale, Boccaccio escogita una soluzione più pratica e collegiale.

Veniamo, allora, all'illustre galleria di exempla: il primo a comparire, per motivi di anzianità, è Andalò del Negro $(6,4)$, "generosus atque venerabilis senex", nonché, "olim", maestro in astronomia dello stesso Boccaccio. Andalò viene introdotto come "familiarissimus" di Ugo IV — secondo le solite implicazioni con il potere - e studioso dall'avanzata metodologia 'empirista', poiché "astrorum motus agnovit, sed, cum universum fere peragrasset orbem, sub quocunque climate, sub quocunque etiam orizonte, experientia discursuum certior factus, visu didicit, quod nos discimus auditu" ("conobbe i moti degli astri, avendo viaggiato quasi per tutto il mondo, sotto qualunque clima e orizzonte; informato dell'esperienza della rivoluzione degli astri, apprese, vedendole, le nozioni che apprendiamo ascoltandole"). La sua autorità viene qui invocata in nome del bisogno di interdisciplinarietà: "circa ea tamen, que ad astra spectare videntur, non aliter quam Ciceroni circa oratoriam aut Maroni circa poeticam exhibendam censeo" ("circa gli argomenti che riguardano gli astri, penso debba essergli data fede, non diversamente che a Cicerone nell'oratoria, o a Virgilio nella poesia"). Il secondo è Dante $(6,5)$, di cui si insiste sugli aspetti professionali: sebbene povero ed esiliato, fu poeta "eruditissimus", dando prova del proprio valore nella Commedia e presso l'università di Parigi ${ }^{38}$. Poi viene citato Francesco da Barberino $(6,6)$, notaio e poeta, esule da Firenze nel 1304, elogiato per l'onestà e la vita morigerata. La quarta personalità di spicco, invece, è Barlaam $(6,7)$, nominato per l'estesa cultura nelle lingua greca, "ut imperatorum et principum Grecorum atque doctorum hominum privilegia haberet”. Segue Paolo da Perugia $(6,8)$, sorta di predecessore di Boccaccio con le Colletiones di argomenti classici e, inoltre, "diu magister et custos bibliothece Roberti, Jerusalem et Sycilie regis incliti" 39 . Il sesto è Leonzio

38 La notizia del falso soggiorno francese di Dante viene introdotta nel Trattatello ( $\$ 74-76)$ come momento felice di studio filosofico e teologico, contrapposto all'affannoso pellegrinaggio da una corte all'altra (Verona, Casentino, Lunigiana, Urbino, Bologna e Padova).

39 Il sovrano viene nominato anche a XIV, 9, 14, in un passo di notevole rilevanza: "Audivi iam dudum illustrem virum Iacobum de Sancto Severino, Tricarici et Clarimontis comitem, dicentem se a patre habuisse suo, Robertum, Karoli regis filium, postea inclitum Ierusalem et Sycilie regem, tam torpentis ingenii puerum fuisse, ut non absque maxima demonstrantis difficultate prima licterarum elementa perciperet, et, cum fere de eo hac in parte amici desperarent omnes, pedagogi ingenium eius, solerti astutia rimantis fabellis Esopi in tam grande studendi sciendique desiderium tractus 
Pilato (6, 9), amico dell'autore e, come Barlaam, "licterarum grecarum doctissimus". Il penultimo è il già ricordato Paolo Dagomari detto Geometra $(6,10)$, "notissimus" a Ugo IV; egli, rinomato a Parigi, in Britannia, Spagna e Africa per gli studi scientifici in aritmetica, geometria e astrologia, sarebbe stato di sicuro un "homo felix", se fosse vissuto in una temperie "liberalior". Chiude l'elenco Francesco Petrarca $(6,11)$, al quale viene concesso uno spazio cospicuo, oltre a occupare l'ultima posizione in climax ascendente: si ricorda l'incoronazione poetica del 1340, l'amicizia con Roberto d'Angiò ${ }^{40}$, l'ottima reputazione goduta all'estero e le opere latine (Africa, Bucolicum carmen, Epistolae, Invective, De vita solitaria e De remediis); ma soprattutto egli funge da guida, "testis", a un'intera generazione: "venerandissimus praeceptor, pater et dominus meus".

$\grave{E}$ agevole trovare, nel denominatore comune delle varie figure, le novitates tanto promesse sembrano avvicinare Boccaccio all'Umanesimo: il riferimento alla sfortuna di Paolo Dagomari, di Dante e di Francesco da Barberino nell'aver operato in un'epoca tanto avara verso gli intellettuali, serve da monito per reagire e dotarsi di strumenti più efficaci rispetto al recente passato. Il momento è talmente insolito che il buon poeta dovrà, pur mantenendo salda la propria autonomia e supremazia, ricorrere anche ad altre discipline. Pare questo l'insegnamento da trarre dalle figure di Andalò e Dagomari, le cui scoperte scientifiche sono ormai imprescindibili nella ricerca del vero, che non si dà quale conquista immutabile, bensì orizzonte cui tendere attraverso il concorso delle varie artes ${ }^{41}$. Ciò andrà

est, ut brevi non tantum domesticas has nobis liberales artes didicerit, verum ad ipsa usque sacre phylosophie penetralia mira perspicacitate transiret; talemque de se fecisse regem, ut a Salomone citra regum nullum doctiorem mortales agnoverint" ("udii già raccontare dall'illustre Jacopo dei Sanseverino, conte di Tricarico e Chiaromonte, che aveva sentito da suo padre che Roberto, il figlio di re Carlo - che fu poi inclito re dei Gerusalemme e di Sicilia — fu da ragazzo così torpido d'ingegno, che non senza gravi difficoltà del precettore poté apprendere i primi elementi delle lettere: e che, mentre tutti gli amici disperavano di lui per questa parte, un pedago go, insinuandosi con le favole di Esopo nel suo animo con solerte astuzia, lo trasse a così gran desiderio di studiare e sapere, che in breve tempo, non solo apprese le arti liberali a noi domestiche, ma anche con mirabile perspicacia passò fino ai penetrali della sacra filosofia e fece di sé un re tale che, eccetto Salomone, gli uomini non ne conobbero alcuno più dotto di lui").

40 La grande stima di Re Roberto per Petrarca è espressa a XIV, 22, 5.

41 In questo Boccaccio mitiga il pregiudizio petrarchesco - e medievale in generale - avvicinandosi alle pratiche che contraddistingueranno l'Umanesimo. Garin (1988: 76) spiega che il celebre insegnante Guarino Veronese (1370- 
accompagnato dall'acquisizione di metodologie innovative, come l'osservazione diretta della realtà praticata da Andalò, che tanto ricorda l'approccio induttivo tipico del Decameron, dalla restituzione dei classici cara a Petrarca e Paolo da Perugia e dallo studio del greco di Barlaam e Leonzio Pilato. Naturalmente simili conquiste non saranno sufficienti se lo scrittore si allontanerà da un comportamento incorruttibile e da uno stile sobrio, personificato in modo esplicito da Francesco da Barberino e Petrarca. Tali virtù consentiranno, come ben rappresentano Dante, Andalò e Petrarca, di intrattenere con il potere un rapporto di collaborazione proficua e bilaterale, senza compromettere l'indipendenza della propria attività.

A ben guardare, le Genealogie sembrano riassumere ogni aspetto elencato e fare sintesi dei consigli esposti, tanto che la vera guida, alla fine dell'opera, non pare più il solo Petrarca. Ne sono fedeli testimoni l'assidua dedizione nella composizione di un testo all'avanguardia e laborioso - che si pone come 'grammatica' dei miti fondativi della letteratura - l'utilizzo di materiali eterogenei - concernenti la mitologia, la storia, la filosofia, la religione pagana e cristiana, l'astronomia, la geografia - nonché la capacità di affrancarsi dal rapporto di committenza. Ma la novitas di cui Boccaccio pare maggiormente fiero riguarda lo studio della lingua e della letteratura greca, materia in cui riesce a sopravanzare il maestro e tracciare un percorso mai battuto. A XV, 7, infatti, lo scrittore spiega perché nell'opera siano mescolati i vocaboli greci con quelli latini. Egli non agisce in base alla mera ostentazione, anzi segue una precisa strategia già umanistica $(7,1)$ : "insipidum est ex rivulis querere, quod possis ex fonte percipere" ("è stolto cercare dai ruscelli ciò che si può attingere dalle fonti") ${ }^{42}$. La convinzione che sia necessario recuperare il greco nasce dalla constatazione dei limiti della cultura coeva $(7,4)$ :

Ast ego in hoc Latinitati compatior, que sic omnino Greca abiecit studia, ut etiam non noscamus caracteres licterarum. Nam, et si sibi suis sufficiat licteris,

Garin (1988: 76) spiega che il celebre insegnante Guarino Veronese (13701460), attivo soprattutto a Ferrara, "legò sempre le 'lettere' alle 'scienze'. Era persuaso che la nuova cultura poteva attuarsi solo in scuole nuove".

42 Vd. anche 7, 3-4: "Volvant, si libet, volumina Ciceronis, videant scripta Macrobii, intueantur Apuleii libros, et, ne plures afferam, Maximi Auxonii opuscula legant; hos sepissime versus Grecos Latinis licteris inserentes invenient. Horum ego vestigia in hoc secutus sum" ""sfoglino i miei critici, se vogliono, le opere di Cicerone o gli scritti di Macrobio; vedano i libri di Apuleio e, per non citarne altri, leggano gli opuscoli di Massimo [ma: Magno] Ausonio; e troveranno che questi spesso hanno inserito versi greci nelle scritture latine. Io ho seguito in ciò le loro norme"). 
et in eas omnis occiduus versus sit orbis, sociate Grecis lucidiores procul dubio apparerent. Nec preterea omnia secum a Grecia veteres traxere Latini, multa supersunt, et profecto nobis incognita, quibus possemus scientes effici meliores ${ }^{43}$.

L'idea di un mondo occidentale, latino e cristiano, che basta solo a sé stesso inizia a essere messa in dubbio, rivendicando sia l'utilità della civiltà greca per conoscere meglio quella latina sia l'urgenza di accedere a un mondo da cui i romani sono stati a lungo dipendenti: le due grandi culle della cultura sono avvertite nella loro ideale unità, da ricomporre a ogni costo. Inoltre queste osservazioni sono adeguate per ribadire ancora una volta la natura del pubblico erudito e dotto cui il testo è rivolto ${ }^{44}$. Ma tutto ciò trova compimento nell'estrema rivendicazione di orgoglio e primogenitura negli studi greci: "si nesciunt, meum est hoc decus mea gloria est, scilicet inter Etruscos Grecis uti carminibus" ("se non lo sanno, questo è mio onore e gloria mia: far uso fra i Toscani di versi greci”). Boccaccio si attribuisce il merito di aver portato in Italia Leonzio Pilato a insegnare greco presso lo Studium fiorentino, ospitandolo e facendolo accettare, sforzo non semplice visto il pessimo carattere di quest'ultimo (cfr. XV, 6, 9), dai colleghi. Inoltre il certaldese afferma di essere stato il primo a ricondurre in Italia le opere omeriche e a farle leggere in pubblico, grazie all'avallo del comune toscano dal 1360 al 1362 . Ecco, quindi, che anche Boccaccio, nel momento in cui espone il massimo motivo di compiacimento per il proprio lavoro, ammette il vincolo fatale di collaborazione con il potere, sempre con un'attenzione particolare, però, alla propria inviolabile libertà $(7,6)$ : "nec in Etruriam tantum, sed in patriam [scil. Homeri

43 "Ma io proprio in questo ho compassione della Latinità del nostro tempo, la quale così totalmente ha rifiutato gli studi greci, che oggi neppure conosciamo i caratteri dell'alfabeto. Se infatti la Latinità sembra bastare a sé e alla sua letteratura, e se tutto il mondo occidentale si è volto ad essa, senza dubbio le lettere latine, se fossero associate alle greche, apparirebbero più splendide. Inoltre gli antichi Latini non hanno tratto tutto il buono dalla Grecia: molte opere restano, e certo a noi sconosciute, conoscendo le quali potremmo diventare più dotti”.

44 Cfr. XV, 7, 5: "Hi demum non prospectant, ad quem hoc opus ego dirigam, cui laborem impendam, vidissent quippe, quoniam eruditissimo regi, et cui tam Grecarum quam Latinarum licterarum, si vera fert fama, notitia est, et quem penes continue docti homines Greci sint, quibus Greca carmina, ut his ignaris, non videbuntur superflua" ("questi critici poi non vedono a chi rivolga quest'opera, per chi spenda la mia fatica; se lo considerassero, vedrebbero che io ho eseguito l'opera a richiesta di un re eruditissimo al quale, se la fama riporta il vero, non sono meno familiari le lettere greche e che le latine, e presso il quale continuamente sono molti dotti greci che non riterranno per superflui, come questi ignoranti, i versi greci”). 
libros] deduxi" ("né solo a Firenze, ma in Italia, li ho ricondotti"). Il richiamo a un compito che soddisfa le richieste politiche contingenti e che bada, allo stesso tempo, a mantenere un respiro universale ci restituisce un impegno consapevole sì dei limiti presenti, ma anche fiducioso nelle straordinarie potenzialità della letteratura.

\section{Università degli Studi di Milano}

\section{OPERE CITATE}

Alighieri, Dante. La Commedia secondo l'antica vulgata, a cura di Giorgio Petrocchi. Milano: Mondadori, 1966-1967.

- Convivio, a cura di Franca Brambilla Ageno. Firenze: Le Lettere, 1995.

—. De Vulgari Eloquentia, a cura di Pier Vincenzo Mengaldo. Padova: Antenore, 1968.

—. Epistole, in Le Opere di Dante, I, a cura di Ermenegildo Pistelli. Firenze: Società Dantesca Italiana, 1960.

-. Quaestio de aqua et terra, in Le Opere..., cit.

—. Vita nuova, a cura di Michele Barbi. Firenze: Bemporad \& Figlio, 1907.

Ascoli, Albert Russell. Dante and the Making of a Modern Author. Cambridge: Cambridge University Press, 2008.

Augustinus, Aurelius. L'istruzione cristiana, a cura di Manlio Simonetti. Milano: Fondazione Lorenzo Valla, 1994.

Avesani, Rino. "Il preumanesimo veronese", in Storia della cultura veneta, II, Il Trecento. Antenore: Vicenza, 1976, 111-141.

Barberi Squarotti, Giorgio. "Le poetiche del Trecento in Italia", in AA. VV. Momenti e problemi di storia dell'estetica, I. Milano: Marzorati, 1959, 303-322.

Bausi, Francesco. Petrarca antimoderno. Studi sulle invettive e sulle polemiche petrarchesche. Firenze: Franco Cesati Editore, 2008.

Bergdolt, Klaus. La peste nera e la fine del Medioevo. Casale Monferrato: Piemme, 1997.

Billanovich, Giuseppe. "Il preumanesimo padovano", in Storia..., cit., 19-110.

Boccaccio, Giovanni, Boccacciós Expositions on Dante's Comedy. A cura di Michael Papio, Toronto: University of Toronto Press, 2009.

Comedia delle ninfe fiorentine, a cura di Vittore Branca, in Tutte le opere di Giovanni Boccaccio, II. Milano: Mondadori, 1964.

. Consolatoria a Pino de Rossi, a cura di Giuseppe Chiecchi, in Tutte le opere..., cit., V, 1994.

—. Decameron, a cura di Vittore Branca. Firenze: Accademia della Crusca, 1976. . De Casibus virorum illustrium, a cura di Pier Giorgio Ricci e Vittorio Zaccaria, in Tutte le opere..., cit., IX, 1983.

. De Mulieribus claris, a cura di Vittorio Zaccaria, in Tutte le opere..., cit., X, 1967.

—. Esposizioni sopra la Comedia di Dante, a cura di Giorgio Padoan, in Tutte le 
opere..., cit., VI, 1965.

- Genealogie deorum gentilium, a cura di Vittorio Zaccaria, in Tutte le opere..., cit., VII-VIII, 1998.

—. Trattatello in Laude di Dante, a cura di Pier Giorgio Ricci, in Tutte le opere..., cit., III, 1974.

—. Vita di Petrarca, a cura di Gianni Villani. Roma: Salerno Editrice, 2004.

Bologna, Corrado. "Dante e il latte delle Muse", in Atlante della letteratura italiana, I, a cura di Amedeo de Vincentiis. Einaudi: Torino, 2010, 145-155.

Branca, Vittore. Boccaccio medioevale. Firenze: Sansoni Editore, 1956.

Cazalé Bérard, Claude. "Boccaccio e la poetica: Mercurio, Orfeo e Giasone, tre chiavi dell'avventura ermeneutica". Studi sul Boccaccio, 22 (1994): 277-306.

Canetti, Luigi. "Boccaccio teologo. Poesia e verità alla fine del Medioevo". Intersezioni, 31 (2011): 179-195.

Cherubini, Giovanni. "La 'crisi del Trecento'. Bilancio e prospettive di ricerca". Studi Storici, 15 (1974): 660-670.

Chines, Loredana. "Petrarca, Boccaccio e le favole antiche". Intersezioni, 31 (2011): 197-206.

Chittolini, Giorgio. La formazione dello Stato regionale e le istituzioni del contado. Secoli XV e XVI. Torino: Einaudi, 1979.

Costantini, Aldo Maria. "Studi sullo zibaldone magliabechiano. III: La polemica con fra Paolino da Venezia”. Studi sul Boccaccio, 10 (1977-1978): 255-275.

Curtius, Ernst Robert. Letteratura europea e Medio Evo latino. Firenze: La Nuova Italia Editrice, 1992.

Dionisotti, Carlo. "Chierici e laici", in Geografia e storia della letteratura italiana. Torino: Einaudi, 1967, 55-88.

—. Discorso sull'Umanesimo italiano, in Geografia..., cit., 179-199.

Dotti, Ugo. Petrarca civile. Roma: Universale Donzelli, 2001.

Frasso, Giuseppe. "Riflessioni sulla 'difesa della poesia' e sul rapporto 'teologia-poesia' da Dante a Boccaccio", in Il pensiero filosofico e teologico di Dante Alighieri, a cura di Alessandro Ghisalberti. Milano: Vita e Pensiero, 2001, 149-173.

Gaeta, Franco. "Letteratura e potere. Dal comune alla corte rinascimentale", in Letteratura italiana. Il letterato e le istituzioni, a cura di Alberto Asor Rosa. Torino: Einaudi, 1982, 149-255.

Gargan, Luciano. "Il preumanesimo a Vicenza, Treviso e Venezia", in Storia..., cit., 142-170.

Garin, Eugenio. "Le favole antiche", in Medioevo e Rinascimento. Bari: Laterza, 1954, 63-84.

—. La cultura del Rinascimento. Milano: Il Saggiatore, 1988.

Genette, Gérard. Soglie. Torino: Einaudi, 1989.

Gilson, Etienne. "Poesie et verite dans la 'Genealogia' de Boccace". Studi sul Boccaccio, 2 (1964): 253-282.

Grousset, René. L'empire du Levant: histoire de la question d'Orient. Paris: Payot, 1946.

Horatius Flaccus, Quintus. Carmina, a cura di Fridrich Klingner. Leipzig: Teubner, 1939. 
Hortis, Attilio. "Le Genealogie degli dei", in Studj sulle opere latine del Boccaccio. Trieste: Libreria Julius Dase Editrice, 1879, 135-219.

Hugo, de Sancto Victore. Didascalicon de studio legendi, a cura di Charles Henry Buttimer. Washington: The Catholic University Press, 1939.

Iori, Giuseppe. "Dante e la poesia", in Atti della Dante Alighieri a Treviso 19962001, III, a cura di Arnaldo Brunello. Società Dante Alighieri: Treviso, 2001, 232-248.

Isidorus, Hispalensis. Etimologie o Origini, a cura di Angelo Valastro Canale. Torino: UTET, 2004.

Klein, Francesca. "Del Buono di Bartolo Niccolò", in Dizionario biografico degli italiani, XXXVI. Roma: Istituto della Enciclopedia italiana, 1988, 377-379.

Kriesel, James C. "The 'Genealogy' of Boccaccio's theory of allegory”. Studi sul Boccaccio, 37 (2009): 197-226.

Martellotti, Guido. "La difesa della poesia nel Boccaccio e un giudizio su Lucano". Studi sul Boccaccio, 4 (1967): 265-279.

Mendez, Sigmund. "La idea dantesca de la poesía como creación alegórica". Medievalia, 32-33 (2001): 57-69.

Mésoniat, Claudio. Poetica theologia. La 'Lucula noctis' di Giovanni Dominici e le dispute letterarie tra '300 e '400. Roma: Edizioni di Storia e Letteratura,1984.

Miglio, Luisa. "Cavalcanti (de Cavalcantibus) Mainardo (Maghinardo)", in Dizionario biografico degli italiani, XXII. Roma: Istituto della Enciclopedia italiana, 1979, 639-640.

Moudarres, Andrea. "Dante, Omero e la 'gloria' della poesia”. Intersezioni, 26 (2006): 287-299.

Padoan, Giorgio. "Mondo aristocratico e mondo comunale nell'ideologia e nell'arte di Giovanni Boccaccio". Studi sul Boccaccio, 2 (1964): 81-216.

Paoli, Marco. La dedica. Storia di una strategia editoriale. Lucca: Maria Pacini Fazzi Editore, 2009.

Papio, Michael. "Boccaccio: mythographer, philosopher, theologian", in Boccaccio in America, a cura di Elsa Filosa e Michael Papio. Ravenna: Longo Editore, 2012, 123-142.

Penco, Gregorio. "Il Trecento", in Storia della Chiesa in Italia, I: Dalle origini al Concilio di Trento. Milano: Jaca Book, 1978, 414-482.

Pépin, Jean. "Allegoria", in Enciclopedia dantesca, I. Roma: Istituto della Enciclopedia italiana, 151-165.

Persius Flaccus, Aulus. Saturae, a cura di Otto Jahn. Leipzig: Teubner, 1843.

Petrarca, Francesco. Familiarium rerum libri, a cura di Ugo Dotti. Torino: Nino Aragno Editore, 2007.

—. Rerum senilium libri, a cura di Ugo Dotti. Torino: Nino Aragno Editore, 2007.

—. Trionfi, a cura di Vinicio Pacca e Laura Paolino. Milano: Mondadori, 1996.

Petrucci, Armando. Libri, scrittura e pubblico nel Rinascimento. Bari: Laterza, 1979.

Romano, Ruggiero. Storia dell'economia italiana, I: Il Medioevo dal crollo al trionfo.

Torino: Einaudi, 1990.

Ronconi, Giorgio. Le origini delle dispute umanistiche sulla poesia: Mussato e 
Petrarca. Roma: Bulzoni, 1976.

Russell, J. Stephen (a cura di). Allegoresis. The craft of allegory in medieval literature. New York-London: Garland, 1988.

Seneca, Lucius Annaeus. De brevitate vitae, a cura di Alfonso Traina. Loescher: Torino, 1970.

_ Epistulae morales ad Lucilium, a cura di Leighton Durham Reynolds. Oxford: Clarendon Press, 1965-1966.

Spera, Francesco. La poesia forte del poema dantesco. Firenze: Franco Cesati Editore, 2010.

Stefanelli, Ruggiero. Boccaccio e la poesia. Napoli: Loffredo Editore, 1978.

Tanfani, Leopoldo. Nicola Acciaiuoli: studi storici. Le Monnier: Firenze, 1863.

Tateo, Francesco. Retorica e poetica fra Medioevo e Rinascimento. Bari: Adriatica Editrice, 1960.

Terzoli, Maria Antonietta (a cura di). I margini del libro. Atti del Convegno internazionale di studi, Basilea (21-23 novembre 2002). Roma-Padova: Antenore, 2004.

Tufano, Ilaria. "I sonetti gnomici contro l'avaritia e la commendatio poesis", in "Quel dolce canto". Letture tematiche delle "Rime" di Boccaccio. Firenze: Franco Cesati Editore, 2007, 147-183.

Vasoli, Cesare. La filosofia medievale. Milano: Feltrinelli, 1961.

Weiss, Roberto. The Renaissance Discovery of Classical Antiquity. Oxford: Blackwell, 1969.

Witt, Ronald G. "Un poeta laureato: Albertino Mussato”, in Atlante..., cit., 134139. 